\title{
Androidal Fat Dominates in Predicting Cardiometabolic Risk in Postmenopausal Women
}

\author{
O. A. Matvienko, ${ }^{1}$ D. L. Alekel, ${ }^{2}$ S. N. Bhupathiraju, ${ }^{2}$ H. Hofmann, ${ }^{3}$ L. M. Ritland, ${ }^{2}$ \\ M. B. Reddy, ${ }^{2}$ M. D. Van Loan, ${ }^{4}$ and C. D. Perry ${ }^{2}$ \\ ${ }^{1}$ School of Health, Physical Education and Leisure Services, 133 Wellness/Recreation Center, University of Northern Iowa, \\ Cedar Falls, IA 50614-0241, USA \\ ${ }^{2}$ Food Science and Human Nutrition, Human Nutritional Sciences Building, Iowa State University, Ames, IA 50011-1123, USA \\ ${ }^{3}$ Department of Statistics, 2413 Snedecor/2624C Howe, Iowa State University, Ames, IA 50011, USA \\ ${ }^{4}$ Western Human Nutrition Research Center, University of California, Davis, CA 95616, USA
}

Correspondence should be addressed to O. A. Matvienko, oksana.matvienko@uni.edu

Received 14 September 2010; Revised 4 November 2010; Accepted 24 November 2010

Academic Editor: Christina Chrysohoou

Copyright ( $) 2011$ O. A. Matvienko et al. This is an open access article distributed under the Creative Commons Attribution License, which permits unrestricted use, distribution, and reproduction in any medium, provided the original work is properly cited.

\begin{abstract}
We hypothesized that soy isoflavones would attenuate the anticipated increase in androidal fat mass in postmenopausal women during the 36-month treatment, and thereby favorably modify the circulating cardiometabolic risk factors: triacylglycerol, LDLC, HDL-C, glucose, insulin, uric acid, C-reactive protein, fibrinogen, and homocysteine. We collected data on 224 healthy postmenopausal women at risk for osteoporosis (45.8-65 y, median BMI 24.5) who consumed placebo or soy isoflavones (80 or $120 \mathrm{mg} / \mathrm{d}$ ) for 36 months and used longitudinal analysis to examine the contribution of isoflavone treatment, androidal fat mass, other biologic factors, and dietary quality to cardiometabolic outcomes. Except for homocysteine, each cardiometabolic outcome model was significant (overall $P$-values from $\leq .0001$ to .0028 ). Androidal fat mass was typically the strongest covariate in each model. Isoflavone treatment did not influence any of the outcomes. Thus, androidal fat mass, but not isoflavonetreatment, is likely to alter the cardiometabolic profile in healthy postmenopausal women.
\end{abstract}

\section{Introduction}

Menopause is associated with an increase in intra-abdominal fat [1], which is considered a major risk factor of atherosclerotic cardiovascular disease (CVD) [2]. However, whether an increased risk of CVD in postmenopausal women is due to altered body composition, changes in reproductive hormones, or some other physiological process associated with menopause has not been clearly established. It is also uncertain as to what extent androidal fat mass may influence CVD risk factors in healthy nonobese women. The risk of chronic disease is perceived to be considerably higher in obese compared to normal weight adults. However, Gautier et al. [3] recently reported that the effect of waist circumference, reflecting androidal fat mass, in increasing the risk for diabetes was more profound among men and women with a normal body mass index (BMI) of less than
$25 \mathrm{~kg} / \mathrm{m}^{2}$ than among those with above normal BMI. Thus, it appears that efforts to reduce abdominal fat accumulation would be beneficial regardless of BMI category.

This study was ancillary to the Soy Isoflavones for Reducing Bone Loss (SIRBL) study, a randomized, doubleblind, placebo-controlled multicenter (Iowa State University (ISU) and University of California at Davis (UCD)) clinical trial funded by the National Institutes of Health (NIH) [4]. We examined contributors (androidal fat mass, duration of menopause, isoflavone treatment, family history of CVD, and dietary quality) to cardiometabolic risk in primarily normal and overweight healthy postmenopausal women. Cardiometabolic outcomes included circulating low-density lipoprotein cholesterol (LDL-C), high-density lipoprotein cholesterol (HDL-C), triacylglycerol, glucose, homeostatic model assessment (HOMA) of insulin resistance, uric acid, $\mathrm{C}$-reactive protein (CRP), fibrinogen, and homocysteine 
(Hcy). We hypothesized that consumption of soy isoflavones in tablet form would attenuate the anticipated increase in central fat (androidal) mass during the 36 months of treatment, which in turn may favorably modify circulating concentrations of cardiometabolic risk factors. We also hypothesized that androidal fat mass, other biologic factors, and dietary quality would influence these cardiometabolic risk factors, but that androidal fat mass would predominate.

\section{Materials and Methods}

2.1. Overall Study Design. The parent study examined the effect of two doses ( 80 versus $120 \mathrm{mg} /$ day) of soy proteinderived isoflavone versus placebo tablets for 36 months on bone loss in healthy postmenopausal women (45 to 65 years of age) who were at risk for osteoporosis. The parent study included power analysis on the primary outcome (lumbar spine bone mineral density) in the methodology and has been previously reported [4]. This ancillary project focused on the relationship between body composition and cardiometabolic risk factors. Many of these cardiometabolic risk factors were tested throughout the study (LDL-C, HDLC, TAG, glucose, and uric acid) whereas some were only tested through the 12 month time point (HOMA, CRP, fibrinogen, and Hcy). The respective Institutional Review Boards (IRB) at ISU (ID no. 02-199) and at UCD (ID no. 200210884-2) approved our study protocol, consent form, and all participant-related materials. Approvals for the dual-energy X-ray absorptiometry (DXA) procedures were obtained from each institution's IRB and State Department of Public Health in Iowa and California. At prebaseline, each participant received a detailed explanation of the study verbally and in writing before signing an informed consent form.

2.2. Participant Recruitment, Screening, and Selection. We recruited (2003 to 2005) women throughout the state of Iowa and in the greater Sacramento and Bay Area regions in northern California primarily through direct mailing lists, stories in local newspapers, and local/regional radio advertisements. Responders $(N=5,255)$ were initially screened via telephone to identify healthy women (without diseases or conditions, not taking hormones or medications) $\leq 65$ years who had undergone natural menopause (cessation of menses 1 to 10 years), were not experiencing excessive vasomotor symptoms, nonsmokers (not currently smoking and who had not smoked in the past 6 months), and had a BMI between 18.5 through 29.9 (except for 9 women from UCD who did not meet the range of inclusion criterion: 8 women had BMI values that ranged from 30 to 32.7 and one woman had a BMI of 17.8, but were enrolled because they were deemed healthy).

The parent SIRBL project established the inclusion/exclusion criteria. We excluded vegans and high alcohol consumers ( $>7$ servings/week), as well as those who were diagnosed with chronic disease, had a first-degree relative with breast cancer, or who chronically used medication (current: cholesterol-lowering and/or antihypertensive; past
3 months: antibiotics; past 6 months: estrogen/progestogen creams, calcitonin; past 12 months: oral hormones/estrogen or selective estrogen receptor modulators; ever: bisphosphonates).

Women who met the initial screening criteria $(N=677)$ were invited to the clinic for further eligibility screening, including BMD assessment using DXA. The SIRBL project focused on disease prevention rather than treatment; thus, women with BMD lumbar spine (L1-L4) and/or proximal femur T-scores that were low ( $>1.5 \mathrm{SD}$ below young adult mean) or high (>1.0 SD above mean) or with evidence of previous or existing spinal fractures were excluded. Once each woman qualified based on BMD, fasting blood was drawn for a clinical chemistry profile. We excluded women with evidence of diabetes mellitus (fasted glucose $\geq 6.93 \mathrm{mmol} / \mathrm{L}(126 \mathrm{mg} / \mathrm{dl})$ ), abnormal renal, liver (elevated enzymes), and/or thyroid function, or elevated lipids (LDL-cholesterol $>4.10 \mathrm{mmol} / \mathrm{L}(160 \mathrm{mg} / \mathrm{dl})$; triacylglycerol $>2.25 \mathrm{mmol} / \mathrm{L}(200 \mathrm{mg} / \mathrm{dl}))$. Based upon our entry criteria, we randomized 255 women to treatment in the parent trial. We excluded 13 women at UCD from this analysis because they did not meet the entry criteria (11 had thickened endometrium, 1 had breast cancer, 1 could not provide a baseline blood sample). We excluded an additional 19 women who did not have body composition data at either 12, 24, or 36 months because they dropped out of the study, resulting in a sample size of 224 women based upon androidal fat as our primary covariate of interest in this ancillary project.

\subsection{Randomization to Treatment and Tablet Formulation.} To meet the objectives of the parent project, participants at each location (ISU, UCD) were stratified according to baseline proximal femur BMD (high, medium, and low) [4] based upon NHANES III database population values [5] and randomly assigned to one of three treatment groups: placebo control, $80 \mathrm{mg}$ isoflavones, or $120 \mathrm{mg}$ isoflavones. Tablets were provided by The Archer Daniels Midland Co. (Decatur, IL); tablet composition has been described previously [4]. An independent researcher (Patricia Murphy) at ISU confirmed that the actual isoflavone doses (mean $\pm \mathrm{SD}, \mathrm{mg} / \mathrm{d}$ ) were similar to those formulated and tested by Archer Daniels Midland, respectively: control $=0$ compared with $0.3 \pm 0.4$; $80 \mathrm{mg}=89.5 \pm 5.0$ compared with $84.3 \pm 4.5 ; 120 \mathrm{mg}=$ $124.0 \pm 7.7$ compared with $122.5 \pm 3.4$. Participants in each group were instructed to take three compressed tablets/d. To preserve the double-blind nature of the study, bottles did not indicate treatment assignment.

\subsection{Body Size and Composition Measures and Blood Sample Measures. For this study, we used anthropometric and body composition measurements that included weight, height, whole body lean and fat mass, and androidal fat mass, as well as fasting concentrations of lipids/lipoproteins, glucose, and uric acid. These outcomes were assessed at baseline, 12, 24, and 36 months. In addition, circulating insulin, HOMA (calculated as fasting glucose $(\mathrm{mg} / \mathrm{dL}) \times$ fasting insulin $(\mu \mathrm{U} / \mathrm{mL}) / 405), \mathrm{CRP}$, fibrinogen, Hcy, and red blood}


cell (RBC) folate concentrations were assessed at baseline and 12 months.

Trained researchers obtained anthropometric measurements according to standard protocols. Standing height was taken twice (average value recorded) with a wall-mounted stadiometer (Model S100; Ayrton Corp., Prior Lake, MN) and weight was measured at ISU using a balance beam scale (ABCO Health-o-Meter; Bridgeview, IL) and at UCD using an electronic scale (Circuits and Systems Inc; E. Rockaway, NY). Women wore hospital scrubs or shorts and a t-shirt, and removed their shoes, belts, watches, and jewelry for the duration of assessment. To ensure standardized data collection, body composition measurements were obtained by certified cross-trained DXA operators using matching DXA instruments (Delphi W Hologic Inc; Bedford, MA) at each site that were calibrated daily. To further ensure quality control, one operator assessed overall composition from the whole body DXA scans for both sites. Regional adiposity analysis was performed by one analyzer (LR) who sectioned each whole body DXA scan into waist, hip, and thigh regions based on bone landmarks [6] using special software (Discovery Version 12.3:7). The waist region included the first lumbar through the fourth lumbar vertebrae. The hip region began below the fourth lumbar vertebrae and extended to the tip of the greater trochanter of the femur. Androidal fat mass $(\mathrm{kg})$ for each participant was the sum of waist and hip fat mass.

Phlebotomists collected fasted $(9 \mathrm{~h})$ blood samples between 7:00 and 8:00 am. We separated serum (allowed to clot for $30 \mathrm{~min}$ prior to centrifugation) and plasma from whole blood and centrifuged samples for 15 minutes $\left(4^{\circ} \mathrm{C}\right)$ at $1000 \times \mathrm{g}$, storing aliquots at $-80^{\circ} \mathrm{C}$ until analyses. Certified clinical laboratories (LabCorp; Kansas City, KS for ISU and UCD Medical Center; Sacramento, CA for UCD) performed a chemistry panel (including serum glucose, lipid profile, and uric acid) on each participant at each time point. We measured the remaining analytes (serum insulin, serum CRP, plasma fibrinogen, plasma Hcy, and RBC folate) for each participant in batch from ISU and UCD samples at baseline and 12 months in duplicate at ISU. We used sufficient in-house sera/plasma as qualitycontrol samples (frozen at $-80^{\circ} \mathrm{C}$ ) to run with each kit to calculate interassay coefficient of variation (CV); we used duplicate samples to calculate intraassay CV. The lowto-normal and normal-to-high controls for each kit were well within the acceptable ranges. Serum insulin $(\mu \mathrm{U} / \mathrm{mL})$ concentration was determined with a radioimmunoassay kit (Linco Research, St Charles, MO, USA) using a Cobra II series autogamma counting system (Packard Instrument Company; Meriden, CT, USA). The intra- and interassay CV for insulin were $3.0 \%$ and $4.0 \%$, respectively. Serum CRP $(\mathrm{mg} / \mathrm{L})$ concentration was determined with a highsensitivity sandwich enzyme-linked immunosorbent assay kit (ALPCO Diagnostics; Salem, NH) and plasma (heparinized) fibrinogen $(\mathrm{mg} / \mathrm{mL})$ concentration was determined with a sandwich enzyme-linked immunosorbent assay kit (AssayPro; St. Charles, MO) using a microtiter plate reader (ELx808; Bio-Tek Instruments, Inc., Winooski, VT). The intra-assay CVs for CRP and fibrinogen were $3.7 \%$ and $2.7 \%$, respectively; the interassay CVs for $\mathrm{CRP}$ and fibrinogen were $6.0 \%$ and $2.3 \%$, respectively. Total $\mathrm{Hcy}(\mu \mathrm{mol} / \mathrm{L})$ concentration was determined using a high-performance liquid chromatography (HPLC) method adapted from Araki and Sako [7] and Ubbink et al. [8]. The total Hcy in plasma consists of free Hcy (i.e., reduced plus oxidized Hcy in the nonprotein fraction of plasma) and protein-bound Hcy [9]. N-Acetylcysteine (1 mM) was added as an internal standard to the plasma samples prior to derivatization. The fluorescence intensities were measured with excitation at $385 \mathrm{~nm}$ and emission at $515 \mathrm{~nm}$, using a JASCO FP1520 fluorescence detector. Further assay details have been previously published [9]. The intra- and interassay CV for plasma Hcy were $3.8 \%$ and $6.3 \%$, respectively. Intracellular (RBC) folate $(\mathrm{ng} / \mathrm{mL})$ was measured using a radioactive immunoassay kit (MP Biomedicals; Irvine, CA). Because four hematocrit values were missing, $\mathrm{RBC}$ folate could not be calculated for these four samples. In addition, three baseline samples were incorrectly processed; thus, RBC folate values have been presented for 217 women. The intraand interassay CV for RBC folate were $3.2 \%$ and $11.8 \%$, respectively.

2.5. Interviewer-Administered Questionnaires. During the enrollment phase, trained interviewers administered three questionnaires to participants: a health and medical history $[10-12]$, a reproductive history [13], and a nutrition history $[10,11]$. We also gathered data on prescription and overthe-counter medications at each time point, as well as previous and/or current use of herbal therapies or dietary supplements (which they were asked to discontinue prior to baseline testing). We assessed dietary intake at each time point using a semiquantitative food frequency questionnaire from Block Dietary Data Systems (Berkeley, CA). A Healthy Eating Index (HEI) score, with higher scores representing greater adherence to federal dietary guidelines, was calculated for each participant based on this questionnaire and included in the statistical analyses as a covariate.

2.6. Statistical Analysis. We performed statistical analyses using version 2.10 .1 of the $\mathrm{R}$ software, including version 3.1.96 of the nlme package software and considered results statistically significant (two sided) at $P \leq .05$. Our 3-year longitudinal analyses of LDL-C, HDL-C, triacylglycerol, and glucose included women with complete data at all time points, baseline through 36 months. Our 1-year longitudinal analyses of fibrinogen, insulin, HOMA, Hcy, and CRP included women with complete data at baseline and 12 months. We reported descriptive statistics for 224 women using median and interquartile range. We constructed longitudinal models to identify significant contributors to each cardiometabolic risk factor (LDL-C, HDL-C, triacylglycerol, uric acid, glucose, insulin, HOMA, CRP, fibrinogen, and $\mathrm{Hcy})$. Each final longitudinal model included these obligatory variables: treatment (control versus combined treatment with $80 \mathrm{mg}$ or $120 \mathrm{mg}$ of isoflavones or, in other words, no treatment versus treatment), time point (baseline versus 12 , 24 , or 36 months), treatment by time point interaction, 
and site (ISU versus UCD), as well as potential covariates that included androidal fat mass $(\mathrm{kg})$ adjusted for height, time since last menstrual period (TLMP) (yr) (calculated for each woman: baseline test date-date of her last menstrual period), family history of CVD coded as a categorical variable (none versus positive or none versus unknown), and HEI score. Additionally, the insulin model included glucose as a covariate, and the Hcy model included RBC folate as a covariate. Independent variables in modeling the outcomes of interest included those variables that were biologically plausible. Preliminary models also included dietary fat intake (determined using the Block Food Frequency Questionnaire) and physical activity (determined using the Paffenbarger physical activity recall [14]), but those variables did not emerge as remotely significant contributors to any of the cardiometabolic risk factors and thus were not included in the final models.

Separate height adjustments of fat and fat-free mass have been suggested for children by Wells and Cole [15]; accordingly, we found a significant impact of height on androidal fat mass among the participants in our study based on a log-log regression analysis (parameter estimate $0.8286, P=.0058$ ). This suggested the use of heightadjusted androidal fat mass (androidal fat mass ( $\mathrm{g}$ )/height $(\mathrm{cm})$ ) (exponent of 0.8286 for height changed to 1 for ease of interpretation). In other words, we adjusted androidal fat mass for height because taller women typically had greater waist circumferences due to their larger frame size and thus appeared to be at higher risk for CVD compared with shorter women, whereas this is not necessarily the case. We performed log transformation of variables with skewed distributions: triacylglycerol, HOMA, CRP, Hcy, and RBC folate.

Restricted maximum likelihood (REML) estimation was used to obtain estimates of variances and correlations between repeated measures. Model selection was guided by a stepwise backwards selection based on model diagnostics, such as Akaike's information criterion and Bayes information criterion. An overall model fit was obtained based on a likelihood ratio test of the (maximum likelihood fitted) model at hand and the more parsimonious model of only obligatory covariates. Significance indicates a failure to accept the null hypothesis of covariates contributing to the model in a random fashion. More specifically, significance indicates that the full model with all of the variables included explains a greater proportion of the variability in the outcome than the parsimonious model.

\section{Results}

3.1. Cardiometabolic Risk Factors Assessed during a 36Month Period. Baseline values for body composition and cardiometabolic outcomes are summarized in Table 1. Body composition measurements, including androidal fat mass, did not change significantly during the course of the study. The isoflavone treatment did not have an effect on any of the body composition outcomes. Treatment compliance was verified using urinary isoflavone concentrations. Compliance was excellent [4]. The results of longitudinal analysis showed that each cardiometabolic outcome model was highly significant, with overall $P$-values $\leq .0001$ for all but the LDL-C model $(P=.002)$. Significant covariates for each analyte are shown in Table 2. Androidal fat mass and site (ISU versus UCD) were consistent predictors of all analytes (36 month data) assessed, including lipids/lipoproteins, glucose, and uric acid. In general, androidal fat mass was typically the strongest covariate (positive) in each model (except the model with HDL-C as the outcome, where it was significantly and negatively associated). Time point also emerged as a significant covariate in the HDL-C and glucose models, indicating that the median concentrations of these analytes increased with time; however, the analyte concentrations at subsequent time points were not significantly different compared to baseline. The median glucose concentration was $85 \mathrm{mg} / \mathrm{dL}$ at baseline and $88 \mathrm{mg} / \mathrm{dL}$ at 36 months. The median HDL-C concentration was $64 \mathrm{mg} / \mathrm{dL}$ at baseline and $65 \mathrm{mg} / \mathrm{dL}$ at 36 months. Family history of CVD had a significant association with glucose: women who were unaware of their family history ("do not know," $n=9$ ), taking into account key factors in the model, had on average a $6.8 \mathrm{mg} / \mathrm{dL}$ higher glucose concentration compared with women who indicated that they had a positive family history. Isoflavone treatment, TLMP, or HEI did not influence any of the 36 month analytes.

3.2. Cardiometabolic Risk Factors Assessed during a 12Month Period. Similar to the 36 month outcomes, each 12 month outcome model was highly significant (overall model $P$-values $\leq .0001)$, except for Hcy $(P=.23$, data not shown). Androidal fat mass was positively associated with each outcome (Table 3 ) and was the strongest covariate (positive) in each model. The parameter estimate for site was negative, indicating that the women from UCD had lower values than the women at ISU. In addition, TLMP $(P=.0020)$ and TLMP-by-site interaction $(P=.0021)$ contributed significantly, and a positive family history of CVD contributed marginally $(P=.070)$ to fibrinogen concentration. As TLMP increased, the difference in fibrinogen concentration between the sites decreased. Glucose and time were significant covariates in the insulin model. As expected, a higher glucose concentration was associated with a higher insulin concentration. The unadjusted mean insulin concentration was significantly greater $(2.86 \mu \mathrm{U} / \mathrm{mL}$; $P \leq .0001)$ at 12 months compared with baseline. Isoflavone treatment or HEI did not emerge as significant predictors in any of the 12 month models.

\section{Discussion}

The main objective of this study was to identify significant contributors to cardiometabolic risk in primarily normal and overweight $(\mathrm{BMI}<30)$ healthy postmenopausal women. In agreement with our hypothesis, androidal fat mass was the strongest and most consistent predictor of all cardiometabolic outcomes (except for Hcy) examined in this study. Our participants, as a group, showed no 
TABLE 1: Characteristics of participants at baseline ${ }^{\mathrm{a}}$.

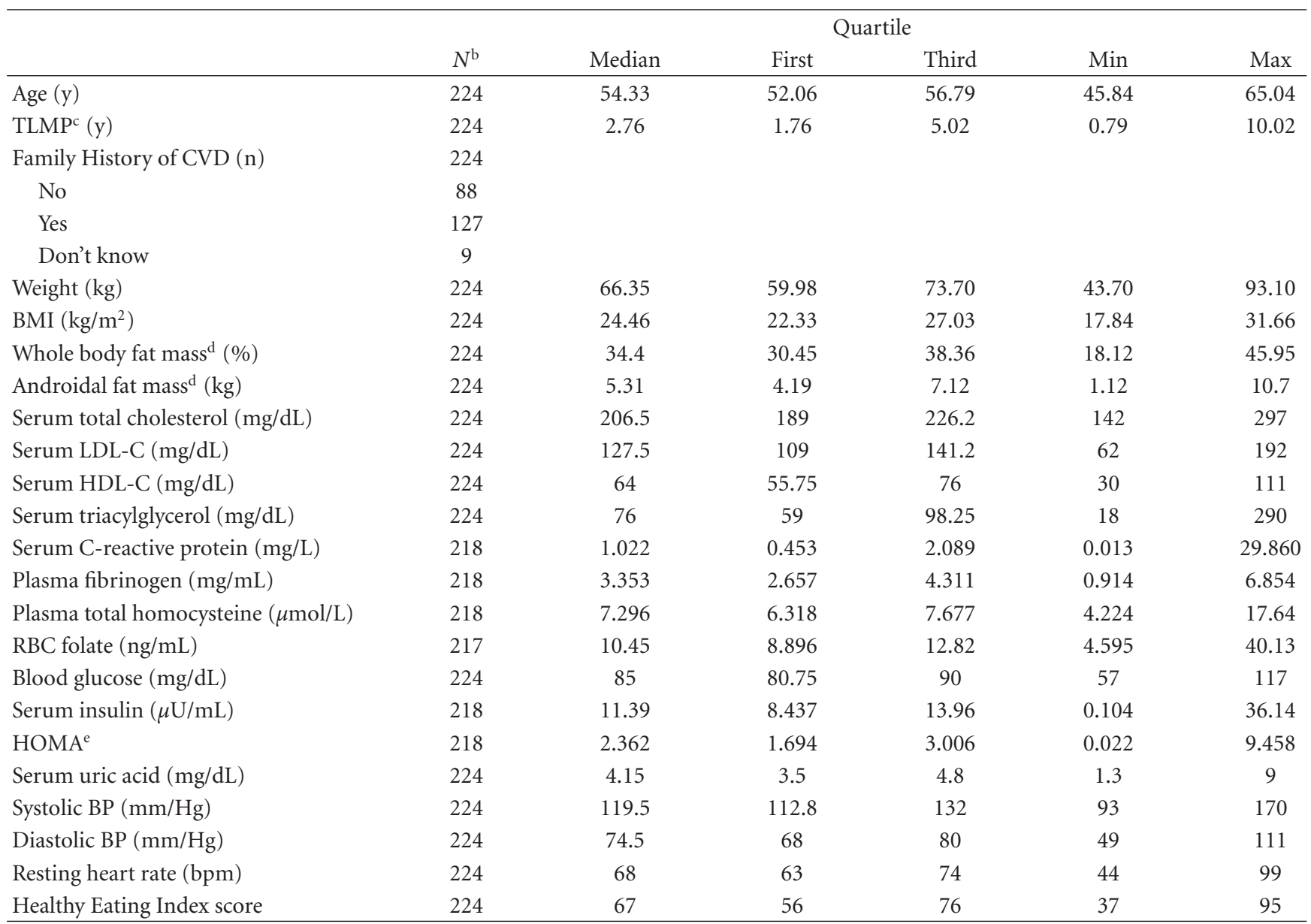

a Baseline data are reported for the entire sample. Treatment groups did not differ significantly in any of the outcomes at baseline.

${ }^{b}$ The number of observations available at baseline

'Time since last menstrual period

${ }^{\mathrm{d}}$ Assessed using dual-energy X-ray absorptiometery (DXA)

${ }^{\mathrm{e}}$ Homeostatic model of insulin resistance

significant changes in body composition measurements, including androidal fat mass, during the course of the study. It should be noted, however, that the women were instructed to maintain their weight throughout the course of the study by following their usual diet and physical activity patterns, and hence, we did not expect to document overall or androidal fat mass change from baseline to 36 months. Similarly, with the exception of insulin, none of the cardiometabolic risk factors changed significantly between baseline and the time of final assessment (either 12 mo or $36 \mathrm{mo}$ ). Nevertheless, strong associations between androidal fat and cardiometabolic risk factors suggested that even a small increase in androidal fat mass may have considerable health consequences. Indeed, Biggs et al. [16] reported that the incidence of type 2 diabetes was 70\% higher (a hazard ratio of 1.7) in adults 65 years of age and older who gained at least $10 \mathrm{~cm}$ in waist circumference over several years compared with those who remained within $2 \mathrm{~cm}$ of their baseline waist circumference. Although the Biggs's study did not specifically target postmenopausal women, it provided some insight into the magnitude of the impact of central adiposity on the incidence of type 2 diabetes.

Certain factors such as duration of menopause, isoflavone treatment, and diet quality that are recognized in the literature as influential with respect to cardiovascular health did not emerge as significant predictors of the cardiometabolic outcomes examined in this study. Some of our findings are consistent with previous reports. For instance, DeNino et al. [17] found that the relationship between age and blood lipids in nonobese women was abolished after controlling for visceral fat. Similarly, in our study, TLMP did not emerge in any model (except for fibrinogen) as a significant covariate, because time point took precedence over TLMP. Results also suggested that androidal fat mass in combination with other factors predominated in these cardiometabolic risk models. On the other hand, our findings for isoflavone treatment and dietary quality conflict with some of the previously published reports. The lowering effect of soy protein rich in isoflavones on blood lipids/lipoproteins, albeit modest 
TABLE 2: Longitudinal analysis: covariates associated with each outcome variable assessed from baseline through 36 months $(N=224)$.

\begin{tabular}{|c|c|c|c|c|c|c|}
\hline Outcome variable & $\begin{array}{l}\text { Independent } \\
\text { variable }\end{array}$ & Likelihood ratio $^{\mathrm{b}}$ & Parameter estimate & Std. error & $t$ value & $\operatorname{Pr}(>t)$ \\
\hline \multirow[t]{2}{*}{ LDL-C } & Androidal fat mass & 8.93 & 0.274 & 0.092 & 2.99 & 0.0029 \\
\hline & Site & & 16.102 & 2.649 & 6.08 & $\leq 0.0001$ \\
\hline \multirow[t]{5}{*}{ HDL-C } & Androidal fat mass & 41.13 & -0.337 & 0.052 & -6.51 & $\leq 0.0001$ \\
\hline & Site & & -6.819 & 1.941 & -3.51 & 0.0005 \\
\hline & Time point 12 & & 1.479 & 1.065 & 1.39 & 0.17 \\
\hline & Time point 24 & & 2.106 & 1.066 & 1.98 & 0.049 \\
\hline & Time point 36 & & 3.607 & 1.066 & 3.38 & 0.0008 \\
\hline \multirow[t]{2}{*}{ Triacylglycerol } & Androidal fat mass & 73.19 & 0.006 & 0.001 & 8.79 & $\leq 0.0001$ \\
\hline & Site & & -0.045 & 0.019 & -2.37 & 0.019 \\
\hline \multirow[t]{5}{*}{ Glucose } & Androidal fat mass & 36.13 & 0.165 & 0.031 & 5.28 & 0.0032 \\
\hline & Site & & -2.310 & 0.894 & -2.59 & 0.010 \\
\hline & Time point 12 & & 2.802 & 1.015 & 2.76 & 0.0059 \\
\hline & Time point 36 & & 3.599 & 1.015 & 3.55 & 0.0004 \\
\hline & $\begin{array}{l}\text { FamHx “don’t } \\
\text { know” }\end{array}$ & & 6.868 & 2.303 & 2.98 & 0.0032 \\
\hline \multirow[t]{2}{*}{ Uric Acid } & Androidal fat mass & 49.30 & 0.027 & 0.004 & 7.19 & $\leq 0.0001$ \\
\hline & Site & & 0.427 & 0.112 & 3.81 & 0.0002 \\
\hline
\end{tabular}

${ }^{a}$ Each final longitudinal model included obligatory variables: treatment (control versus combined treatment with $80 \mathrm{mg}$ or $120 \mathrm{mg}$ of isoflavones), time point (baseline versus 12, 24, or 36 months), treatment by time point interaction, and site (ISU versus UCD), as well as potential covariates that included androidal fat mass (kg) adjusted for height, time since last menstrual period (TLMP), family history of CVD (Fam Hx) coded as a categorical variable (none versus positive or none versus unknown), and Healthy Eating Index (HEI) score. This table shows only those covariates that were significant $(P \leq .05)$ for each outcome variable or had a tendency $(P \leq .10)$ to be significant.

${ }^{b}$ The likelihood ratio for each model represents the ratio of a model that includes only obligatory variables compared to the final model that includes obligatory variables and covariates. The overall $P$ value for each model was $\leq .0001$, except the LDL model had a $P$ value $=.0028$.

( $\leq 6 \%$ reduction), has been documented $[18,19]$. The effect is more profound in hypercholesterolemic individuals and in males. Similarly, the effect of diet quality on CVD outcomes is well known: a healthier diet (high in fiber and low in fat and sodium) is associated with a lower risk of CVD $[20,21]$. We included both a HEI score as a measure of diet quality and isoflavone treatment in our analysis. None of the models retained either covariate. The lack of association between HEI and cardiometabolic outcomes in our study could be in part explained by a majority of women who had relatively healthy diets (median HEI of 67 out of 100). Very few studies have examined the association between HEI and cardiometabolic outcomes with mixed results. For instance, Kant and Graubard [22] reported that HEI emerged as a negative predictor of serum Hcy, CRP and plasma glucose $(P<.05)$, whereas Fung and colleagues [23] did not find an association between HEI and CRP. In a recent study of 125 multiethnic overweight and obese women in early postpartum, the HEI scores were negatively associated with LDL-C and total cholesterol and positively related to HDL-C after adjustment for energy intake, body weight, and lactation status [24].

Based on the results of our study, HEI does not appear to be a predictor of cardiometabolic risk factors. On the other hand, the effects of dietary factors as well as isoflavone treatment on cardiometabolic outcomes may be mediated by androidal fat mass. We previously reported that soy isoflavone treatment for 12 months did not exert an effect on body composition, including androidal fat, in our sample of women [25]. With respect to diet, Fox et al. [26] determined that premenopausal women who participated in a 24 week diet and/or exercise program showed reductions in weight $(\sim 7 \mathrm{~kg})$ and total percentage body fat (although it remained greater than $35 \%$ for all groups), but no significant improvements in blood lipids, glucose, or insulin concentrations. Further analysis revealed a lack of change in the waist-to-hip ratio, which in turn indicated that body fat distribution was not influenced by the intervention. To summarize, dietary interventions that are not sufficiently potent to reduce androidal fat mass are not likely to produce changes in cardiometabolic outcomes.

In addition to androidal fat mass, the other most common independent predictors of cardiometabolic outcomes in our study included time and site. Although concentrations of HDL-C and glucose did not change significantly during the study, both analytes showed an upward trend from baseline to $36 \mathrm{mo}$. The effect of time could be related to biological and/or behavioral factors that were not included in our models. In fact, TLMP was no longer significant when time was included, indicating that time was more important than TLMP. The site variable also emerged as a significant covariate in all models: compared with women at ISU, 
TABLE 3: Multivariate linear regression: covariates associated with each outcome variable assessed from baseline through 12 months.

\begin{tabular}{|c|c|c|c|c|c|c|}
\hline Outcome variable ${ }^{\mathrm{b}}$ & $\begin{array}{l}\text { Independent } \\
\text { variable }\end{array}$ & Likelihood ratio $^{c}$ & Parameter estimate & Std. error & $t$ value & $\operatorname{Pr}(>t)$ \\
\hline \multirow[t]{2}{*}{ CRP } & Androidal fat mass & 90.69 & 0.052 & 0.005 & 24.50 & $\leq 0.0001$ \\
\hline & Site & & -0.236 & 0.121 & 10.79 & 0.072 \\
\hline \multirow[t]{5}{*}{ Fibrinogen } & Androidal fat mass & 31.63 & 0.012 & 0.004 & 4.32 & $\leq 0.0001$ \\
\hline & Site & & -1.469 & 0.227 & -6.48 & $\leq 0.0001$ \\
\hline & TLMP & & -0.118 & 0.038 & -3.13 & 0.0020 \\
\hline & TLMP $\times$ Site & & 0.159 & 0.051 & 3.12 & 0.0021 \\
\hline & Positive Fam Hist & & 0.204 & 0.112 & 1.82 & 0.070 \\
\hline \multirow[t]{4}{*}{ Insulin } & Androidal fat mass & 51.16 & 0.143 & 0.024 & 5.95 & $\leq 0.0001$ \\
\hline & Site & & -4.516 & 0.588 & -7.68 & $\leq 0.0001$ \\
\hline & Time point 12 & & 2.013 & 0.650 & 3.1 & 0.0022 \\
\hline & Glucose & & 0.095 & 0.028 & 3.4 & 0.0008 \\
\hline \multirow[t]{3}{*}{ HOMA } & Androidal fat mass & 43.73 & 0.012 & 0.002 & 6.88 & $\leq 0.0001$ \\
\hline & Site & & -0.312 & 0.039 & -7.92 & $\leq 0.0001$ \\
\hline & Time point 12 & & 0.011 & 0.004 & 3.44 & 0.0007 \\
\hline
\end{tabular}

aThe number of observations included in the analysis varied depending on the outcome: $N=217$ for CRP and Hcy, $N=218$ for fibrinogen, insulin, and HOMA. The Hcy model was not statistically significant and thus not shown in this table.

${ }^{b}$ Each final longitudinal model included these obligatory variables: treatment (control versus combined treatment with 80 mg or 120 mg of isoflavones), time point (baseline versus 12, 24, or 36 months), treatment by time point interaction, and site (ISU versus UCD), as well as potential covariates that included androidal fat mass $(\mathrm{kg})$ adjusted for height, time since last menstrual period (TLMP), family history of CVD (FamHx) coded as a categorical variable (none versus positive or none versus unknown), and Healthy Eating Index (HEI) score. The fibrinogen model included TLMP by site interaction. The insulin model (but not other models) included glucose as a covariate. This table shows only those covariates that were significant $(P \leq .05)$ for each outcome variable or had a tendency $(P \leq .10)$ to be significant.

${ }^{\mathrm{C}}$ The likelihood ratio for each model represents the ratio of a model that includes only obligatory variables compared to the final model that includes obligatory variables and covariates. The overall $P$-value for each model was $\leq .0001$.

women at UCD had lower concentrations of triacylglycerol, HDL-C, glucose, insulin, HOMA, CRP, and fibrinogen and higher concentrations of LDL-C and uric acid. The site effect may be in part explained by baseline characteristics: ISU women were slightly (albeit not significantly) heavier and younger compared with UCD women. It is also quite possible that the site effect was due to some inherent differences between the two geographic locations. We did not document a relationship between androidal fat mass, as well as other factors, and Hcy likely because the women in our study were well below the clinical cut-off of 15, and Hcy values did not indicate great variability.

Some of the strengths of our study were that we followed a relatively large number of women and monitored longitudinal changes (baseline to 12 or $36 \mathrm{mo}$ ) in cardiometabolic risk factors. Limitations were that these women were free-living (both a strength and limitation), lacked ethnic diversity, and underwent infrequent measurements (yearly). Women in this study were mainly nonobese based upon the BMI definition. However, BMI does not adequately reflect body composition. Thus, some of our women may have been identified as obese using other criteria, such as percentage body fat $(35-40 \%=$ overweight, $>40 \%=$ obese [27]). In conclusion, although cardiometabolic outcomes examined in this study were not affected by isoflavone treatment, each outcome (except for Hcy) had a strong, significant association with androidal fat mass. Thus, even small changes in androidal fat are likely to alter the cardiometabolic profile in healthy postmenopausal women.

\section{Conflict of Interests/Financial Disclosures}

Coauthors have not reported conflicts or disclosures.

\section{Funding}

The SIRBL clinical trial was supported mainly by a Grant (RO1 AR046922) from the National Institute of Arthritis and Musculoskeletal and Skin Diseases (NIAMS). The project was also supported by the Nutrition and Wellness Research Center, Iowa State University; USDA/ARS, Western Human Nutrition Research Center, Clinical and Translational Science Center, Clinical Research Center at the University of California (1M01RR19975-01), and National Center for Medical Research (UL1 RR024146). Archer Daniels Midland Company (Decatur, IL) donated soy isoflavone tablets (Novasoy) and GlaxoSmithKline (Moon Township, PA) donated calcium and vitamin D supplements (Os-Cal).

\section{Acknowledgments}

Study concept and design; secured funding: Alekel, Matvienko, Van Loan. Acquisition of data: Alekel, Bhupathiraju, Matvienko, Perry, Ritland, Van Loan. Study supervision: Alekel, Van Loan. Administrative, technical support: Alekel, Van Loan. Statistical analysis and support: Hofmann. Analysis and interpretation of data: Alekel, Hofmann, Matvienko. Drafting of paper: Matvienko. Critical revision of paper for important intellectual 
content: Alekel, Bhupathiraju, Hofmann, Matvienko, Reddy. Final approval of paper: Alekel, Bhupathiraju, Hofmann, Matvienko, Perry, Reddy, Ritland, Van Loan. The SIRBL study team would like to thank all of our participants, since without their dedication, our study could not have been completed. The authors would like to acknowledge our phlebotomists and students (graduate and undergraduate alike) who reported early and steadfastly for testing at our clinic sites. The authors thank the James R. Randall Research Center, Archer Daniels Midland Company (Decatur, IL) that supplied free-of-charge the ingredients, using certified good manufacturing procedures, for the treatment tablets (Novasoyâ), as well as Atrium Biotechnologies Inc. that compressed the ingredients into tablets. The authors thank GlaxoSmithKline (Moon Township, PA) for donating the calcium and vitamin D supplements (Os-Cal). The authors would also like to thank our DSMB (Dennis Black, Ph.D., Chair) and Joan McGowan, Ph.D., Director, Musculoskeletal Diseases Branch at NIAMS, who provided scrutiny, guidance, and valuable feedback throughout the trial.

\section{References}

[1] M. J. Toth, A. Tchernof, C. K. Sites, and E. T. Poehlman, "Menopause-related changes in body fat distribution," Annals of the New York Academy of Sciences, vol. 904, pp. 502-506, 2000.

[2] P. Mathieu, P. Pibarot, É. Larose, P. Poirier, A. Marette, and J. P. Després, "Visceral obesity and the heart," International Journal of Biochemistry and Cell Biology, vol. 40, no. 5, pp. 821-836, 2008.

[3] A. Gautier, R. Roussel, P. H. Ducluzeau et al., "Increases in waist circumference and weight as predictors of type 2 diabetes in individuals with impaired fasting glucose: influence of baseline BMI — data from the DESIR study," Diabetes Care, vol. 33, no. 8, pp. 1850-1852, 2010.

[4] D. L. Alekel, M. D. Van Loan, K. J. Koehler et al., "The Soy Isoflavones for Reducing Bone Loss (SIRBL) Study: a 3-y randomized controlled trial in postmenopausal women," The American Journal of Clinical Nutrition, vol. 91, no. 1, pp. 218230, 2010.

[5] A. C. Looker, H. W. Wahner, W. L. Dunn et al., "Updated data on proximal femur bone mineral levels of US adults," Osteoporosis International, vol. 8, no. 5, pp. 468-489, 1998.

[6] L. M. Ritland, D. L. Alekel, O. A. Matvienko et al., "Centrally located body fat is related to appetitive hormones in healthy postmenopausal women," European Journal of Endocrinology, vol. 158, no. 6, pp. 889-897, 2008.

[7] A. Araki and Y. Sako, "Determination of free and total homocysteine in human plasma by high-performance liquid chromatography with fluorescence detection," Journal of Chromatography, vol. 422, pp. 43-52, 1987.

[8] J. B. Ubbink, W. J. H. Vermaak, and S. Bissbort, "Rapid highperformance liquid chromatographic assay for total homocysteine levels in human serum," Journal of Chromatography, vol. 565, no. 1-2, pp. 441-446, 1991.

[9] S. N. Bhupathiraju, D. L. Alekel, J. W. Stewart et al., "Relationship of circulating total homocysteine and C-reactive protein to trabecular bone in postmenopausal women," Journal of Clinical Densitometry, vol. 10, no. 4, pp. 395-403, 2007.
[10] D. L. Alekel, A. ST. Germain, C. T. Peterson, K. B. Hanson, J. W. Stewart, and T. Toda, "Isoflavone-rich soy protein isolate attenuates bone loss in the lumbar spine of perimenopausal women," The American Journal of Clinical Nutrition, vol. 72, no. 3, pp. 844-852, 2000.

[11] D. L. Alekel, Contributions of physical activity, body composition, age, and nutritional factors to total and regional bone mass in premenopausal aerobic dancers and exercisers, Ph.D. dissertation, University of Illinois-Urbana, Urbana, Ill, USA, 1993.

[12] A. Morabia and M. C. Costanza, "International variability in ages at menarche, first livebirth, and menopause. World Health Organization Collaborative Study of Neoplasia and Steroid Contraceptives," American Journal of Epidemiology, vol. 148, no. 12, pp. 1195-1205, 1998.

[13] A. St Germain, C. T. Peterson, J. G. Robinson, and D. L. Alekel, "Isoflavone-rich or isoflavone-poor soy protein does not reduce menopausal symptoms during 24 weeks of treatment," Menopause, vol. 8, no. 1, pp. 17-26, 2001.

[14] R. S. Paffenbarger Jr., A. L. Wing, and R. T. Hyde, "Physical activity as an index of heart attack risk in college alumni," American Journal of Epidemiology, vol. 108, no. 3, pp. 161-175, 1978.

[15] J. C. K. Wells and T. J. Cole, "Adjustment of fat-free mass and fat mass for height in children aged $8 \mathrm{y}$," International Journal of Obesity, vol. 26, no. 7, pp. 947-952, 2002.

[16] M. L. Biggs, K. J. Mukamal, J. A. Luchsinger et al., "Association between adiposity in midlife and older age and risk of diabetes in older adults," Journal of the American Medical Association, vol. 303, no. 24, pp. 2504-2512, 2010.

[17] W. F. Denino, A. Tchernof, I. J. Dionne et al., "Contribution of abdominal adiposity to age-related differences in insulin sensitivity and plasma lipids in healthy nonobese women," Diabetes Care, vol. 24, no. 5, pp. 925-932, 2001.

[18] S. Zhan and S. C. Ho, "Meta-analysis of the effects of soy protein containing isoflavones on the lipid profile," The American Journal of Clinical Nutrition, vol. 81, no. 2, pp. 397408, 2005.

[19] K. Taku, K. Umegaki, Y. Sato, Y. Taki, K. Endoh, and S. Watanabe, "Soy isoflavones lower serum total and LDL cholesterol in humans: a meta-analysis of 11 randomized controlled trials," The American Journal of Clinical Nutrition, vol. 85, no. 4, pp. 1148-1156, 2007.

[20] M. S. K. Lockheart, L. M. Steffen, H. M. Rebnord et al., "Dietary patterns, food groups and myocardial infarction: a case-control study," British Journal of Nutrition, vol. 98, no. 2, pp. 380-387, 2007.

[21] E. P. López, C. Rice, D. O. Weddle, and G. J. Rahill, "The relationship among cardiovascular risk factors, diet patterns, alcohol consumption, and ethnicity among women aged 50 years and older," Journal of the American Dietetic Association, vol. 108, no. 2, pp. 248-256, 2008.

[22] A. K. Kant and B. I. Graubard, "A comparison of three dietary pattern indexes for predicting biomarkers of diet and disease," Journal of the American College of Nutrition, vol. 24, no. 4, pp. 294-303, 2005.

[23] T. T. Fung, M. L. McCullough, P. K. Newby et al., "Diet-quality scores and plasma concentrations of markers of inflammation and endothelial dysfunction," The American Journal of Clinical Nutrition, vol. 82, no. 1, pp. 163-173, 2005.

[24] B. S. Shah, J. H. Freeland-Graves, J. M. Cahill, H. Lu, and G. R. Graves, "Diet quality as measured by the healthy eating index and the association with lipid profile in low-income women in 
early postpartum," Journal of the American Dietetic Association, vol. 110, no. 2, pp. 274-279, 2010.

[25] O. A. Matvienko, D. L. Alekel, U. Genschel, L. Ritland, M. D. Van Loan, and K. J. Koehler, "Appetitive hormones, but not isoflavone tablets, influence overall and central adiposity in healthy postmenopausal women," Menopause, vol. 17, no. 3, pp. 594-601, 2010.

[26] A. A. Fox, J. L. Thompson, G. E. Butterfield, U. Gylfadottir, S. Moynihan, and G. Spiller, "Effects of diet and exercise on common cardiovascular disease risk factors in moderately obese older women," The American Journal of Clinical Nutrition, vol. 63, no. 2, pp. 225-233, 1996.

[27] D. Gallagher, S. B. Heymsfield, M. Heo, S. A. Jebb, P. R. Murgatroyd, and Y. Sakamoto, "Healthy percentage body fat ranges: an approach for developing guidelines based on body mass index," The American Journal of Clinical Nutrition, vol. 72, no. 3, pp. 694-701, 2000. 


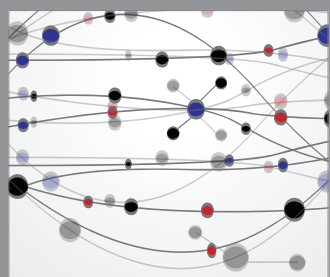

The Scientific World Journal
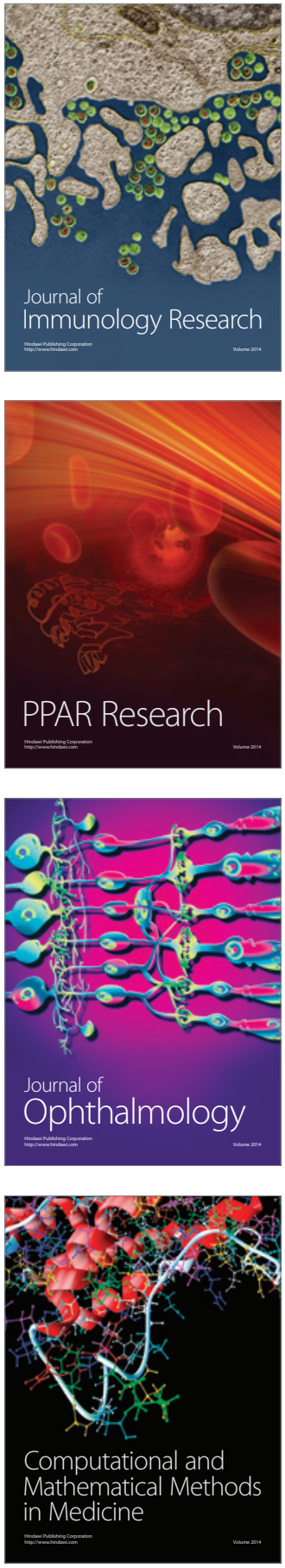

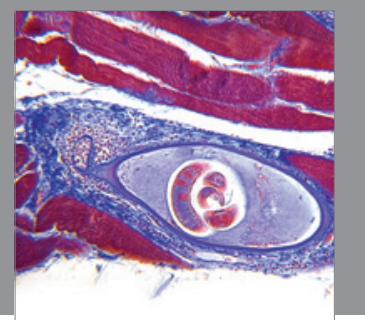

Gastroenterology

Research and Practice
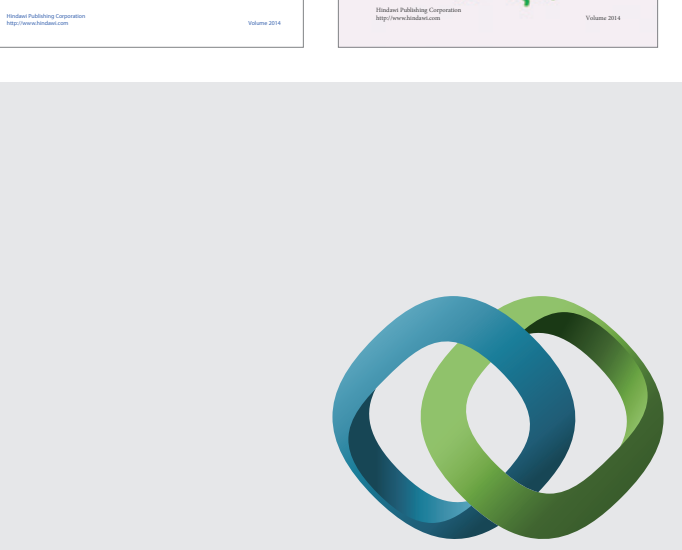

\section{Hindawi}

Submit your manuscripts at

http://www.hindawi.com
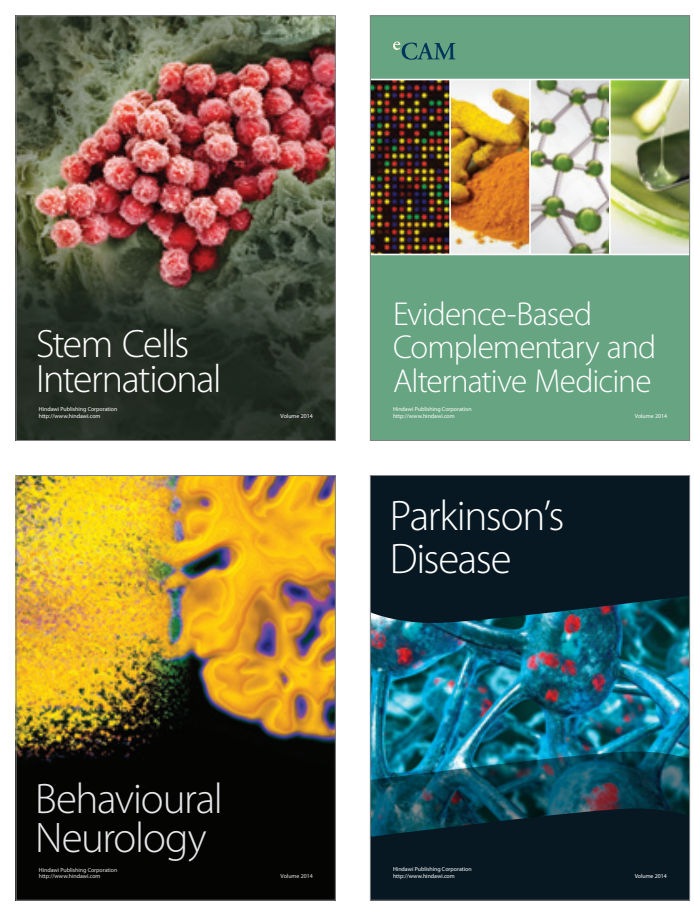

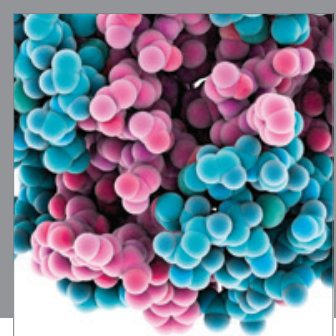

Journal of
Diabetes Research

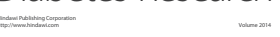

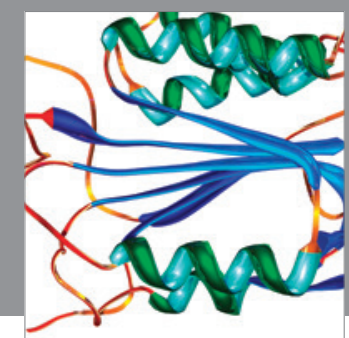

Disease Markers
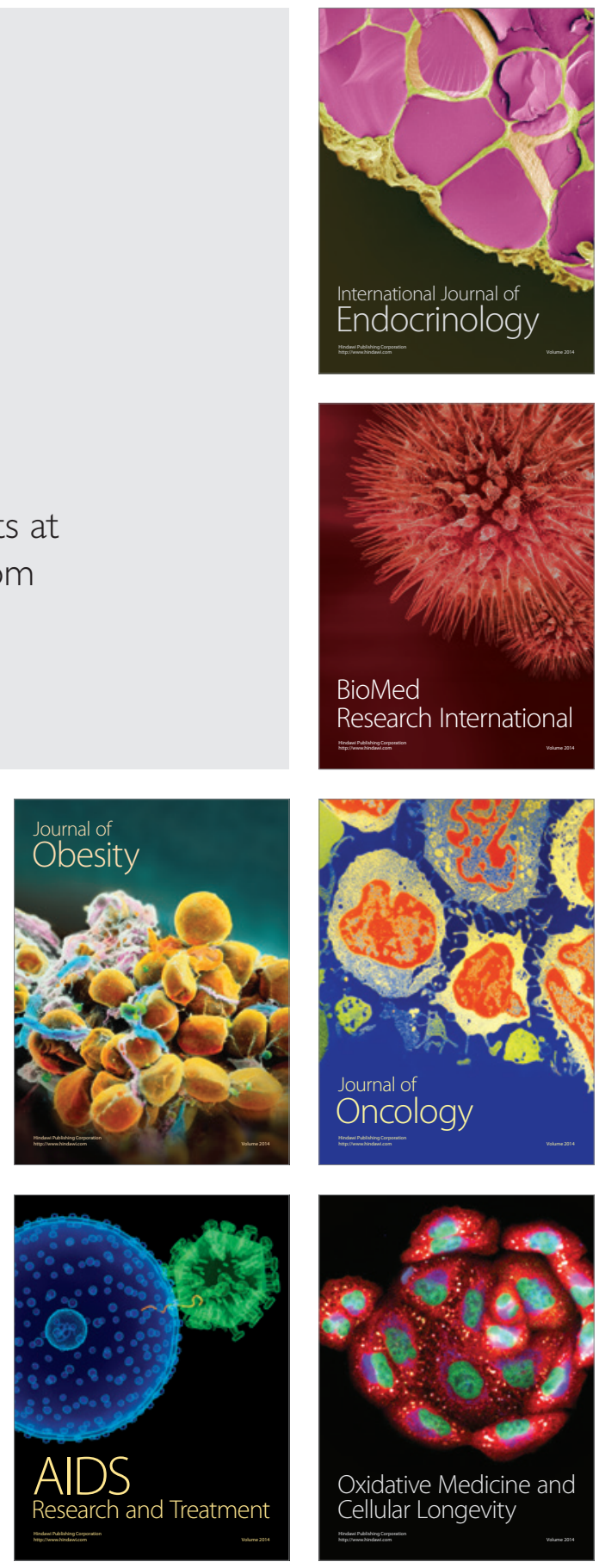